\title{
Incidental Detection of Breast Apocrine Carcinoma via Endometrial Cytology
}

\author{
Mitsuru Shiota, ${ }^{1}$ Yasushi Kotani, ${ }^{1}$ Masahiko Umemoto, ${ }^{1}$ Takako Tobiume ${ }^{1}$ and \\ Hiroshi Hoshiai ${ }^{1}$
}

${ }^{1}$ Department of Obstetrics and Gynecology, Kinki University Faculty of Medicine, Osaka, Japan

\begin{abstract}
Breast apocrine carcinoma is a rare malignancy characterized histologically by a predominance of acidophilic tumor cells exhibiting apocrine metaplasia. Apocrine tumors represent only about $0.4 \%$ of all breast cancers. Many cases are diagnosed at stage I or II; thus, the prognosis is better than that for other types of breast cancer. Here we present a 57-year-old female patient with primary apocrine breast carcinoma that was incidentally discovered by endometrial cytology. The patient had undergone routine uterine cancer screening at the age of 56 with resulting positive endometrial cytology. Subsequent histological examination of an endometrial biopsy revealed suspected metastatic uterine adenocarcinoma. Further evaluation revealed a 3-cm mass in the left breast, which was histopathologically diagnosed as breast cancer. Dual procedures were performed at the departments of gynecology and breast surgery. The patient underwent a modified radical mastectomy of the left breast and left axillary lymph node dissection. Laparotomy revealed multiple adhesions throughout the peritoneal cavity that suggested invasive metastatic cancer, and a right adnexectomy was performed. Histopathological examination of the resected left breast and right ovary tissues revealed apocrine carcinoma. Thus, stage IV breast cancer was diagnosed. The patient finished 6 cycles of paclitaxel and is now under observation on an outpatient basis. In this patient, breast cancer was discovered incidentally through a detailed work-up after a positive result of endometrial cytology. Uterine cancer screening has the potential, although rare, to detect metastatic cancer originating elsewhere. This should be taken into consideration during the work-up due to positive endometrial cytology.
\end{abstract}

Keywords: apocrine carcinoma; breast cancer; endometrial cytology; metastasis; uterine cancer screening Tohoku J. Exp. Med., 2011, 225 (4), 235-237. C 2011 Tohoku University Medical Press

Apocrine carcinoma of the breast is a rare type of breast cancer. It is estimated to represent $0.4 \%$ of all breast cancers (Mossler et al. 1980). Many of the cases are diagnosed at stage I or II; thus the prognosis is thought to be better than that for other breast cancers (Gădăleanu and Galatâr 1986). The 10-year survival rate has been reported to be $52 \%$ (d'Amore et al. 1988).

Metastatic foci of breast cancers are often detected in the lungs, liver, bones, or ovaries. To the best of our knowledge, there has been only one report on breast cancer that was detected from an initial diagnosis of a uterine metastasis, although it was not an apocrine carcinoma of the breast (Kumar and Hart 1982).

Here, we report a patient with apocrine carcinoma of the breast with uterine metastasis that was diagnosed through evaluation of the patient because of positive endometrial cytology.

\section{Patient}

A 57-year-old woman, gravid 2 para 2, postmenopausal for 6 years, with no remarkable past medical history or family history, had a diagnosis of negative for intraepithelial lesion or malignancy (NILM) with cervical cottonswab scraping cytology and positive endometrial cytology on a routine uterine cancer screen at the age of 56. She was referred to our hospital for detailed examination and treatment. The initial pelvic examination revealed a normalsized uterus in anteversion and anteflexion with poor mobility, nonpalpable bilateral adnexa, erosion of the vaginal portion of the cervix, and white discharge. Cervical cottonswab scraping cytology was NILM and endometrial cytology was positive (Fig. 1). Subsequent histology of the endometrium revealed an unequivocal metastatic uterine adenocarcinoma (Fig. 2). A work-up was immediately performed. Results of an upper gastrointestinal contrast study, colonofiberoscopy, and abdominal ultrasonography were

Received June 21, 2011; revision accepted for publication October 20, 2011. doi: 10.1620/tjem.225.235

Correspondence: Mitsuru Shiota, Department of Obstetrics and Gynecology, Kinki University Faculty of Medicine, 377-2 Ohno-higashi,

Osaka-sayama, Osaka 589-8511, Japan.

e-mail: shiota@med.kindai.ac.jp 
A.

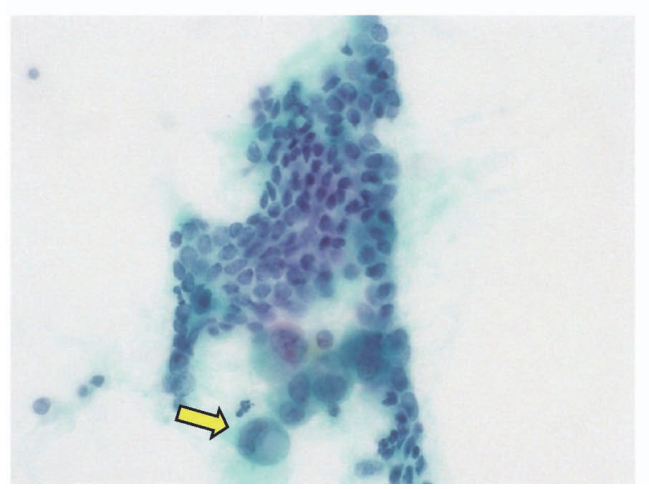

B.

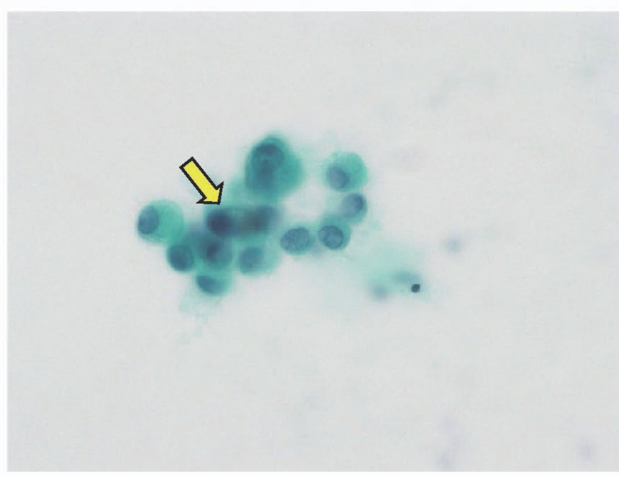

Fig. 1. Endometrial cytology.

Shown are malignant cells developed as a sheet (A) and a small cluster of malignant cells (B). An arrow in (A) indicates an adenocarcinoma cell with empty cytoplasm cell, and an arrow in (B) indicates a cell-in-cell structure. Heterogeneous nuclei and chromatin condensation are observed in the cell cluster as a whole (Papanicolaou staining, $\times 200$ ). The clear background with no tumor-related finding suggests metastatic cancer.

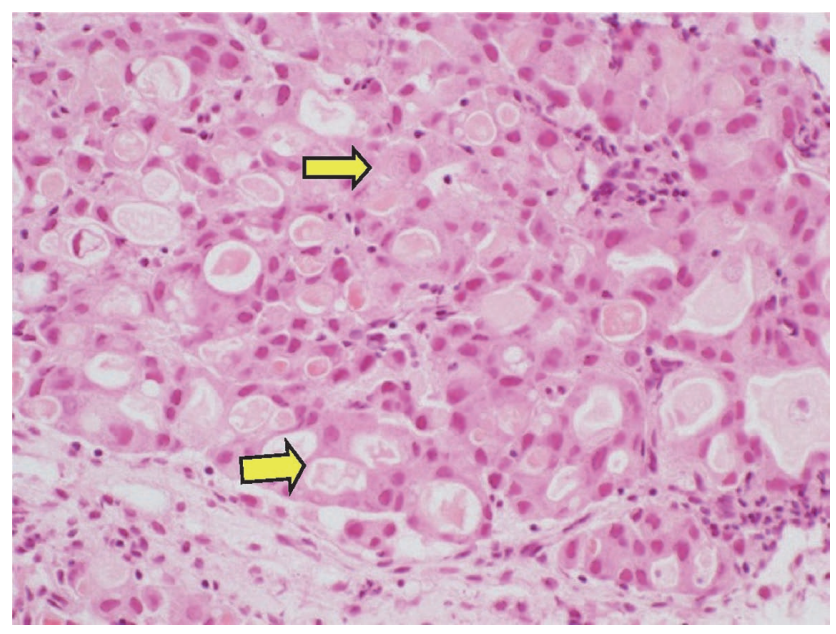

Fig. 2. Endometrial histology.

Heterogeneous nuclei and chromatin condensation are observed in the cytoplasm after hematoxylin and eosin (H\&E) staining $(\times 200)$. In the area indicated by the upper arrow, the round and relatively large cells are observed with relatively small nuclei in which densely stained granules are observed. In the area indicated by the lower arrow, retained fluid substance is clearly observed in the glandular cavity.

unremarkable. Chest computed tomography showed enlargement of the left cervical and the axillary lymph nodes. Biopsy of a left cervical lymph node revealed poorly differentiated adenocarcinoma. A 3-cm mass was found in the left breast, and histopathological examination of a biopsy specimen revealed a possible scirrhous carcinoma of the breast. ${ }^{67} \mathrm{Ga}$-citrate scintigraphy showed accumulation in the left breast. Carcinoembryonic antigen (CEA) was $6.2 \mathrm{ng} / \mathrm{ml}$ (normal range: 0-5.0). Other serum tumor markers were within normal limits. Carbohydrate antigen (CA) 125 was $7 \mathrm{U} / \mathrm{ml}$ (normal: 0-35); CA19-9 was $8 \mathrm{U} / \mathrm{ml}$ (normal 0-37); sialyl Tn antigen (STN), $16.6 \mathrm{U} / \mathrm{ml}$ $(<35)$; tissue polypeptide antigen (TPA), $25 \mathrm{U} / \mathrm{ml}(<70)$; $\alpha$-fetoprotein (AFP), $3 \mathrm{ng} / \mathrm{ml}(<10)$; protein induced by vitamin $\mathrm{K}$ absence-2 (PIVKA-II), $19 \mathrm{mAU} / \mathrm{ml}(<40)$; nation cancer center-stomach (NCC-ST) -439, $1.1 \mathrm{U} / \mathrm{ml}(<$ 7.0); breast carcinoma antigen (BCA) 225, $56.3 \mathrm{U} / \mathrm{ml}(<$ 160); and CA15-3, $14.9 \mathrm{U} / \mathrm{ml}(<27)$. Based on these results, the diagnoses of breast cancer and endometrial cancer (unequivocal metastatic cancer) were made, and the patient underwent surgery.

\section{Surgical findings and diagnosis}

Dual procedures were performed at the departments of gynecology and breast surgery. The patient underwent a modified radical mastectomy of the left breast and a left axillary lymph node dissection. A $3.5-\mathrm{cm}$ tumor was found in the $\mathrm{C}$ region of the left breast. The tumor plus a $2 \mathrm{~cm}$ margin was removed in addition to the fascia of the pectoralis major. The patient then underwent laparotomy. Intraperitoneal findings included a uterus of normal size with poor mobility, a swollen, club-shaped right fallopian tube, and strong adhesions between the suspensory and proper ligaments of the ovary and peritoneum. Invasive metastatic cancer involving the uterus and right adnexa was suspected and hysterectomy was judged difficult. The abdomen was closed after right adnexectomy and collection of peritoneal lavage fluid. The surgery took 4 hours 40 minutes, with a blood loss of $912 \mathrm{~g}$.

By post-operative pathology, apocrine carcinoma and invasive carcinoma were diagnosed from the left breast tissue. The surgical margins were clear of any cancer cells. All 27 excised axillary lymph nodes showed metastatic findings. Apocrine carcinoma was also detected from right ovarian tissue. Peritoneal lavage fluid was positive.

The patient was diagnosed with stage IV breast cancer, has finished 6 cycles of paclitaxel, and is now under observation on an outpatient basis. 
A.

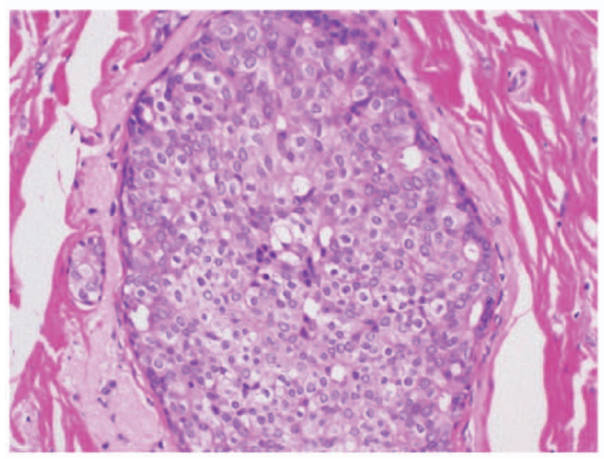

B.

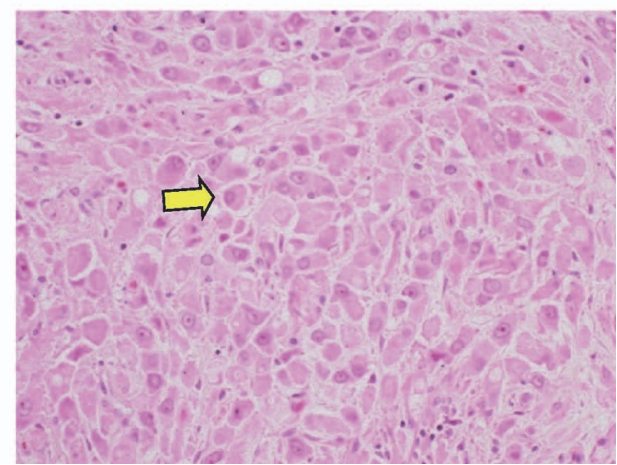

Fig. 3. Breast tissue and ovarian tissue.

A. Breast tissue. Apocrine granules, heterogeneous nuclei and chromatin condensation are observed in the entire breast tissue sample $(H \& E$ staining, $\times 200)$. B. Ovarian tissue. An arrow indicates an apocrine metaplasic cell with a relatively small nucleus in the ovarian tissue sample $(H \& E$ staining, $\times 200)$.

\section{Discussion}

Apocrine carcinoma of the breast is classified as an infiltrating special-type carcinoma, and dominance of apocrine metaplasia is required for the diagnosis. It is a rare histological type; its reported prevalence is $0.4 \%$ of all breast cancers (Mossler et al. 1980). The prognosis is said to be relatively good compared to other breast cancers (Gădăleanu and Galatâr 1986). By contrast, some apocrine carcinomas of the skin are reported to be extremely malignant and rapidly progress and metastasize (Morris et al. 1986). Therefore, it is thought that some apocrine carcinomas of the breast may be extremely aggressive.

Among the gynecological organs, the ovary is the most frequent site of metastasis from breast cancers. It has been reported that $30 \%-38 \%$ of metastatic ovarian cancers originate from breast cancers (Webb et al. 1975; Demopoulos et al. 1987; Gagnon and Têtu 1989). It is thought to be extremely rare for breast cancer to metastasize only to the uterus, and most uterine metastases are thought to occur through ovarian metastasis (Lamovec and Bracko 1991). Although a hysterectomy could not be performed, this patient was thought to have developed a uterine metastasis through an ovarian metastasis, since apocrine carcinoma was detected in the excised right adnexa.

To the best of our knowledge, there has only been 1 report on breast cancer that was detected from an initial diagnosis of a uterine metastasis, although it was not an apocrine carcinoma of the breast (Kumar and Hart 1982). In this case, breast cancer was discovered incidentally as a result of a detailed work-up after a positive result from uterine cancer screening. Uterine cancer screening has the potential, although it may happen rarely, to detect metastatic cancer originating elsewhere. This should be taken into consideration during work-up of a positive uterine cancer screening test.

\section{Conflict of Interest}

All authors have no conflict of interest in this study.

\section{References}

D’Amore, E.S., Terrier-Lacombe, M.J., Travagli, J.P., Friedman, S. \& Contesso, G. (1988) Invasive apocrine carcinoma of the breast: a long term follow-up study of 34 cases. Breast Cancer Res. Treat., 12, 37-44.

Demopoulos, R.I., Touger, L. \& Dubin, N. (1987) Secondary ovarian carcinoma: a clinical and pathological evaluation. Int. J. Gynecol. Pathol., 6, 166-175.

Gădăleanu, V. \& Galatâr, N. (1986) Apocrine carcinoma of the breast. Morphol. Embryol. (Bucur), 32, 33-38.

Gagnon, Y. \& Têtu, B. (1989) Ovarian metastases of breast carcinoma. A clinicopathologic study of 59 cases. Cancer, 64, 892-898.

Kumar, N.B. \& Hart, W.R. (1982) Metastases to the uterine corpus from extragenital cancers. A clinicopathologic study of 63 cases. Cancer, 50, 2163-2169.

Lamovec, J. \& Bracko, M. (1991) Metastatic pattern of infiltrating lobular carcinoma of the breast: an autopsy study. J. Surg. Oncol., 48, 28-33.

Morris, D.M., Sanusi, I.D. \& Lanehart, W.H. (1986) Carcinoma of eccrine sweat gland: experience with chemotherapy, autopsy findings in a patient with metastatic eccrine carcinoma, and a review of the literature. J. Surg. Oncol., 31, 26-30.

Mossler, J.A., Barton, T.K., Brinkhous, A.D., McCarty, K.S., Moylan, J.A. \& McCarty, K.S. Jr. (1980) Apocrine differentiation in human mammary carcinoma. Cancer, 46, 2463-2471.

Webb, M.J., Decker, D.G. \& Mussey, E. (1975) Cancer metastatic to the ovary: factors influencing survival. Obstet. Gynecol., 45, 391-396. 